\title{
Research on Phase Detection of Liquid Crystal Optical Device
}

\author{
Mingliang Shi, Ruofu Yang, Chunping Yang, Mingwu Ao, Hongzhou Dong \\ School of Optoelectronic Information, University of Electronic Science and Technology of China, Chengdu, \\ China \\ Email: shimingliang083@126.com
}

Received July 2014

\begin{abstract}
The technology for phase detection of liquid crystal optical device is a difficult research in current domestic and overseas. However, for the existing liquid crystal optical device, aiming at the poor anti-vibration capability and poor versatile of phase detection, the complexity of phase retrieval algorithm, we propose a new phase measurement principle and experimental methods of liquid crystal optical device. It is a phase measurement method based on the combination of phaseshifting interferometer and phase conjugation technology. The deflection characteristics of the liquid crystal device means the device can implement phase modulation to only one direction of polarized light while is completely transparent to orthogonal polarized light. We put forward the phase shift of the orthogonal polarization phase shift interferometer method, using phase shifting interference as well as the combination of phase conjugate means to achieve its phase measurement. So we can retrieves devices modulation phase simply and efficiently combines with phaseshifting interferometer technology.
\end{abstract}

\section{Keywords}

Liquid Crystal Optical Device, Modulation Phase Detection, Orthogonal Phase Shifting, Conjugate Interference, Phase Retrieval Algorithm

\section{Introduction}

Fast development of the liquid crystal optical device makes miniature, inexpensive adaptive optics, space optical communication systems become possible. Such optics for accurate quantification of the incident wave front phase modulation capability relates to the ability to realize the above-mentioned applications and is very hard to be measured and quantified accurately restricted by measurement method. There are many detection methods for modulation phase of liquid crystal retarders [1]-[4]. For Liquid Crystal Spatial Light Modulator and LC-lens optical devices, double-slit interference method and Mach-Zehnder interferometer measurements have some limitations [5]-[8]. And to require higher stability of the detector and put higher requirements on the processing of the optical element [9] [10]. Some improved measurements such as the common optical path radial shearing interferometry, conjugate phase-shifting interferometry [11] [12], or their phase retrieval algorithm is complex, iteration is slow, or can not accurately assess the Wave front phase of the exit end of the liquid crystal, and lost 
high-frequency phase seriously.

We reference the methods for liquid crystal phase grating in 1996 Raytheon company by L. J. Friedman and others [13]. Using the polarization properties of the liquid crystal optical device making signal light and reference light improvement of the common optical path, which enhances the next earthquake resistance and reduce the sensitivity of the long optical path of the airflow, and thus realize the system structure of quasi-optical path.

\section{The Phase Detection Principle Based on Orthogonal Phase Shifting Conjugated Technology for Liquid Crystal Optical Device}

L. J. Friedman and others put forward the modulation phase detection optical path of liquid crystal grating based on Phase-imaging shown in Figure 1.

Because of the interference structure of the method used to make the signal light and the reference light is not common path, so poor vibration resistance, especially in the long optical path. In this paper, a reference to this method and has been improved, so that it has better anti-interference ability.

The improved structure was shown in Figure 2. The detection system consists of laser 1, a laser beam expander mirror 2 , the half-wave plate 3 , the polarization splitting prism 4 , a piezoelectric ceramic reflector 5, 1/4 wave plate 6,7 , a mirror 8 , a transmissive liquid crystal device 9 , the conjugate optical system 10 , the analyzer 11, plane array detectors 12 and LCD panel drive 13 components.

The modulation phase detection system based on transmissive liquid crystal optical device works as follows. The polarization direction of the linearly polarized laser light emitted by the laser 1 is controlled by the half-wave plate 3, through the polarization splitting prism 4, then Split into two beams. The transmitted light $\mathrm{P}$ through the $1 / 4$ wave plate 6 ( $x$-axis and the fast axis make the angle of $45^{\circ}$ ) turn into the left-handed circularly polarized light, which through the mirror 8 reflected back to 1/4 wave plate 6 and becomes s, then s polarized light passes through the polarization splitting prism 4, after again reflected off the beam path which is a reference arm; Another route polarization reflected by the polarization splitting prism 4, through the $1 / 4$ wave plate 7 ( $x$-axis and the fast axis make angle of $45^{\circ}$ ), then pass a piezoelectric ceramic reflector 5 , which is reflected back and through the $1 / 4$ wave plate 7 then turn into $p$, then $p$ polarized light is transmitted through the polarization splitting prism 4 , this road is the signal beam arm. Submicron movement was accurately generated by the piezoelectric ceramic reflector 5 from the signal beam arm, and which generated orthogonal linear polarization phase shift. For transmissive liquid crystal device 9, make the liquid crystal molecular in long axis direction or the direction of polarization parallel with s polarized. When the LCD panel drive 13 make voltage is applied to a transmissive liquid crystal device 9 , the rotation would be happed in such a case which the liquid crystal molecular long axis direction of electric field. so that the incident light $s$ was introduced an additional phase shift ,but the incident light $p$ not. There by achieving a different modulation of the two orthogonal polarizations of light. Collecting the interference fringe by the plane array detectors 12, and use phase-shifting interferometry technology recovery of the liquid crystal modulation phase.

The modulation phase detection system based on transmissive liquid crystal optical device shown in Figure 3, We can see the detection system consists of the laser 1 , a laser beam expander mirror 2, the half-wave plate 3 , the polarization splitting prism 4 , a piezoelectric ceramic reflector $5,1 / 4$ wave plate $6,1 / 4$ wave plate7, a mirror 8, the LCD panel driver 9, the reflective liquid crystal device 10, conjugate optical system 11, an analyzer 12 , plane array detectors 13 and the beam splitter 14 .

The modulation phase detection system based on reflective liquid crystal optical device compares with a phase modulation and transmission type liquid crystal optical, we could see that orthogonal phase shifting conjugated technology working principle of the optical path in the front part they were similar. Except that in front of the reflective liquid crystal device 10, we put into the beam splitter 14. Using the conjugate optical system 11, a reflective liquid crystal device 10 in exit end with the plane array detectors 13 constitute a conjugate image plane, to improve the interference pattern extraction, beam splitter 14 incident plane introduced AR coated on surface, sub-transmission surface film introduced beam-splitting film, and the antireflection coating request the transmittance deviation of $s$ polarized light was smaller than $p$ polarized light.

\section{Research on Modulation Phase Recovery Algorithm}

After the plane array detector obtained the interference fringes, the process is as follows. Modulation phase recovery algorithm consists of three parts: a liquid crystal layer wrapped phase modulation recovery algo- 


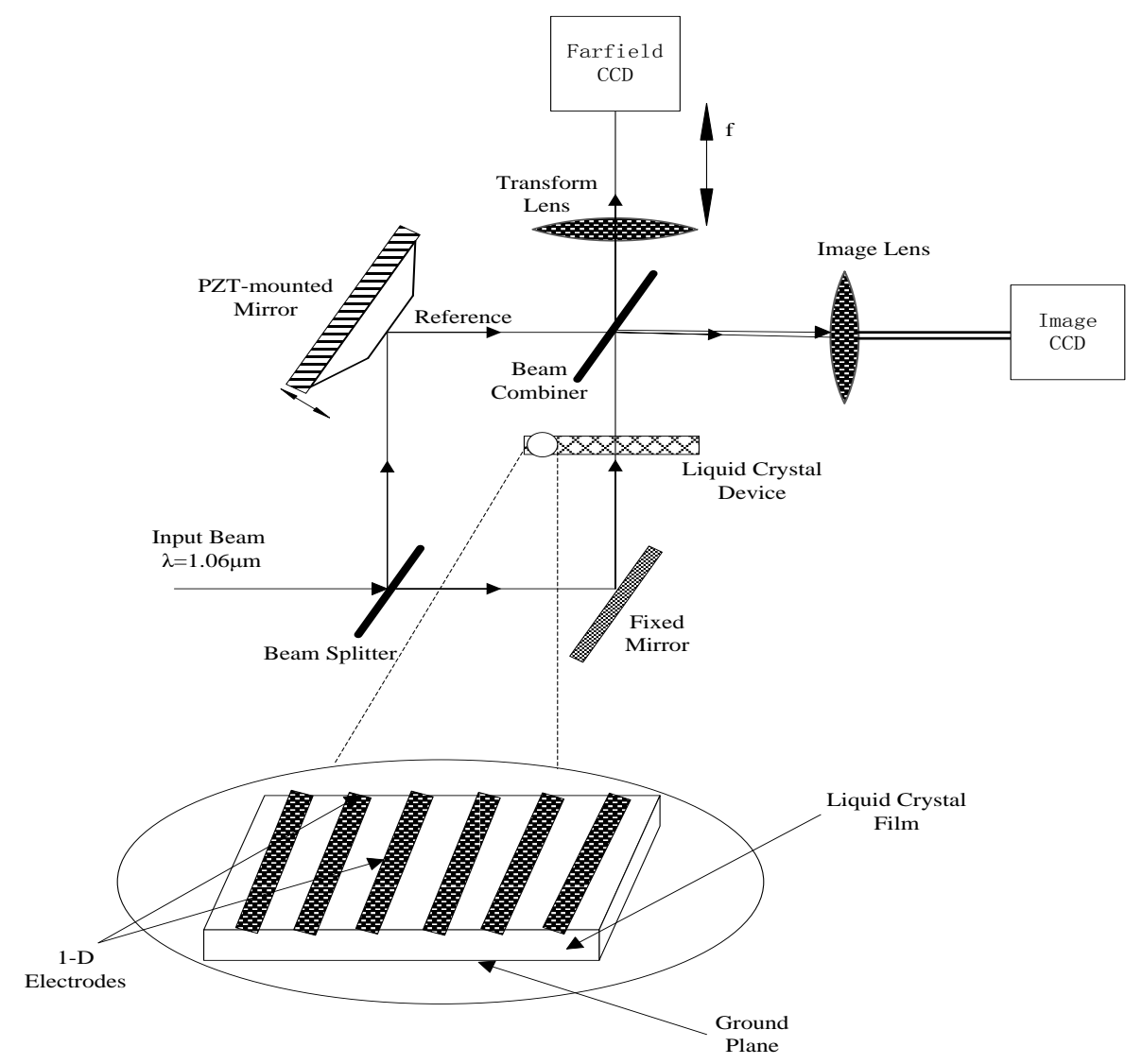

Figure 1. The modulation phase detection optical path of liquid crystal grating based on Mach-Zehnder interferometer.

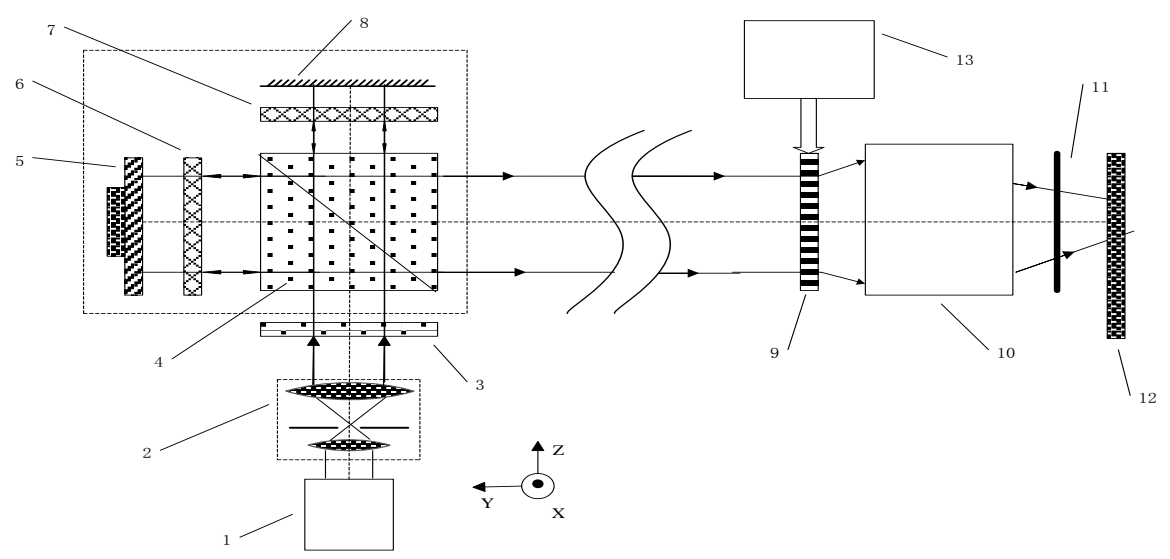

Figure 2. Modulation phase detection system based on transmissive liquid crystal optical device.

rithm, unwrapping algorithm and system aberration calibration. Considered in the case of a simplified model of the detection system (for simplicity, only the transmission type liquid crystal optical device is considered to have a phase modulated wave front, and the rest of the detection system is an ideal lens, prism, planar surface), using a polarization optical matrix derive unwrapping algorithm.

Let's assume the Jones vector of the incident light beam was $E_{1}=\left[\begin{array}{l}A_{1} \\ B_{1}\end{array}\right]$, There has a phase modulation for $s$ 


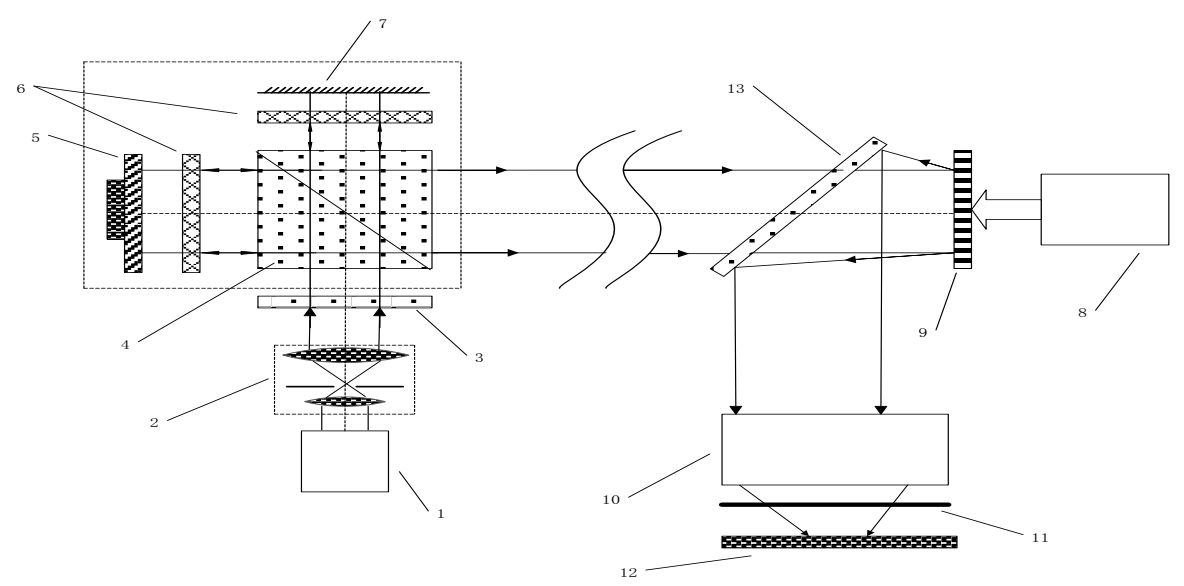

Figure 3. Modulation phase detection system based on reflective liquid crystal optical device.

polarized light, then $p$ polarized light without a phase modulation. For s polarized light, After transmitted by a plurality of optical transmission elements, the Jones vector of the plane array detectors was becoming $E_{1}^{\prime}=\left[\begin{array}{l}A_{1}^{\prime} \\ B_{1}^{\prime}\end{array}\right]$, and:

$$
E_{1}^{\prime}=\prod_{i=1}^{7} J_{8-i} E_{1}
$$

$J_{7}, \cdots, J_{1}$ was the Jones matrix, which respectively represent from the sample analyzer into the half-wave plate, $s$ polarized light through the transmission path of the optical system. $\varphi$ was the liquid crystal phase modulation, $\theta$ was the direction of polarization of the polarizer and the angle between the $X$ axis coordinate system. After deduced:

$$
E_{1}^{\prime}=\prod_{i=1}^{7} J_{8-i} E_{1}=\left[\begin{array}{c}
\frac{A_{1} i}{2} \sin 2 \theta \\
A_{1} \sin ^{2} \theta
\end{array}\right] \mathrm{e}^{i \varphi}
$$

As the same to ppolarized light, after deduced:

$$
E_{2}^{\prime}=\left[\begin{array}{l}
B_{1} i \cos ^{2} \theta \\
\frac{B_{1} i}{2} \sin 2 \theta
\end{array}\right] \mathrm{e}^{i \frac{2 \pi(2 \Delta)}{\lambda}}
$$

So, the amplitude distribution on the plane array detectors:

$$
E^{\prime}=E_{1}^{\prime}+E_{2}^{\prime}=\frac{i}{2}\left[\begin{array}{l}
A_{1} \sin 2 \theta \mathrm{e}^{i \varphi}+2 B_{1} \cos ^{2} \theta \mathrm{e}^{i \frac{2 \pi(2 \Delta)}{\lambda}} \\
2 A_{1} \sin ^{2} \theta \mathrm{e}^{i \varphi}+B_{1} \sin 2 \theta \mathrm{e}^{i \frac{2 \pi(2 \Delta)}{\lambda}}
\end{array}\right]
$$

Commonly, to Simple, we provided with the polarization direction $45^{\circ}$ to the $X$ axis, so we could obtain the distribution of interference intensity was:

$$
I=\left|E^{\prime}\right|^{2}=\frac{1}{4}\left(A_{1}^{2}+B_{1}^{2}\right)+\frac{1}{2} A_{1} B_{1} \cos \left(\varphi-\frac{4 \pi \Delta}{\lambda}\right)
$$

Assuming the amount of movement $\Delta$, driving the piezoelectric mirror reflecting were $0, \lambda / 8, \lambda / 4,3 \lambda / 8$, so we obtain the interference light intensity, then calculated by four interferograms, which we can derive wrapped phase as:

$$
\varphi=\arctan \frac{I_{4}-I_{2}}{I_{1}-I_{3}}
$$

For different liquid crystal device, like a liquid crystal spatial light modulator, a liquid crystal grating, the Liquid crystal phase retarder, etc., their phase modulation's amplitude does not exceed $2 \pi$, so the phase was 
calculated wrapped phase is the phase detection. The liquid crystal lens and a new liquid crystal device such as a liquid crystal optical wedge, which may exceed $2 \pi$, they need for unwrapping the phase [14].

\section{Research Results and Analysis}

In summary, we proposes orthogonal phase shifting conjugated technology for testing the phase modulation of liquid crystal optical device, as phase modulation of the devices is effective for linearly polarized light in one direction and is ineffective in orthogonal direction. We make phase shifting method and orthogonal polarize the incident beam into phase reference arm and signal arm as polarized light in common optical path in order to testing the phase modulation of liquid crystal optical device.

The detection system has a great anti-vibration capability and as simple and efficient as the phase retrievalalgorithm as only a short part of the optical path based on the above measuring principle is non-coaxial, the rest are completely coaxial. Meanwhile, the conjugate optical system realizes the diffraction-type liquid crystal device modulation phase detection.

\section{Conclusion}

We proposes orthogonal phase shifting conjugated technology for testing the phase modulation of liquid crystal optical device and realizes system structure of the optical path, deduces the basic process of the phase detection mathematically, demonstrates the feasibility and effectiveness of this method. The basic principles involved is the phase-shifting interferometer combining with phase conjugation technology, orthogonal polarize the incident beam into phase reference arm and signal arm as polarized light in common optical path in order to testing the phase modulation of liquid crystal optical device. Combined with the liquid crystal phase-shifting interferometer technology, so it easily achieve the recovery of modulation phase. It has great difference with the original non-common optical path detection of optical devices and the complex phase retrieval algorithm. As far as we know, there are no reports at home and abroad.

\section{References}

[1] Oilman, S.E., Baur, T.G., Gallagher, D.J., Optics, M., Longmont, C. and Shankar, N.K. (1989) Properties of Tunable Nematic Liquid Crystal Retarders. SPIE, 1166, 461-471.

[2] Bai, L.L., Li, D. and Huang, Z.Q. (2012) Liquid Crystal Variable Retarders. Solid State Phenomena, 181-182, 293-300.

[3] Hwang, S.J. (2005) Precise Optical Retardation Measurement of Nematic Liquid Crystal Display Using the PhaseSensitive Technique. IEEE Journal of Display Technology, 1, 77-81.

[4] Zhang, Y., Zhao, H.J., Zhou, P.W. and Zhao, H.B. (2009) Photoelectric Characteristics of Liquid Crystal Variable Retarder. Foreign Electronic Measurement Technology, 28, 17-20.

[5] Konforti, N., Marom, E. and Wu, S.-T. (1988) Phase Only Modulation with Twisted Nematie Liquid Crystal Spatial Light Modulation. Optics Letters, 13, 251-254. http://dx.doi.org/10.1364/OL.13.000251

[6] Li, Y., Wang, H. and Jin, H.Z. (2005) A New Approach for Making Kinoform from Hologram. Laser \& Infrared, 35, 765-767.

[7] Chen, H.X., Li, D.H. and Chen, Z.P. (2000) Measuring Phase Modulation Characteristic of LC-SLM by Using PhaseShift Interference. SPIE, 4231, 384-386.

[8] Zhang, Y., Wu, L.Y. and Zhang, J. (2006) Study on the Phase Modulation Characteristics of Liquid Crystal Spatial Light Modulator. Journal of Physics: Conference Series, 48, 790-794.

[9] Zhao, X.F., Li, D.H. and Chen, Z.P. (2002) Measuring Phase Modulation Characteristics of Liquid Crystal Spatial Modulators by Using Cyclic Radial Shearing Interference. Journal of Sichuan University, 39, 671-675.

[10] Mcmanamon, P.F., Dorschner, T.A., Corkum, D.L., Friedman, L.J., Hobbs, D.S., Holz, M., Liberman, S., Nguyen, H.Q., Resler, D.P., Sharp, R.C. and Watson, E.A. (1996) Optical Phased Array Technology. Proceeding of IEEE, 84, 268-298.

[11] Zheng, C.Y. and Wu, J. (2009) Measuring the Wavefront Distortion of a Phased-Array Laser Radar by Using a RealTime Optoelectronic Measurement System. Proceeding of SPIE, 7511, 75111T-1-75111T-8.

[12] Zheng, C.Y., Yang, R.F., Xu, L., Kong, L.J., Ao, M.W., Yang, P., Huang, Z.Q. and Wu, J. (2011) Wave-Front Measurement Approach of Liquid-Crystal Blazed Gratings. Chinese Journal of Lasers, 38, 181-186.

[13] Friedman, L.J., Hobbs, D.S., Lieberman, S., Corkum, D.L., Nguyen, H.Q., Resler, D.P., Sharp, R.C. and Dorschner, 
T.A. (1996) A Spatially Resolved Phase Imaging of a Programmable Liquid-Crystal Grating. Applied Optics, 35, 62366240. http://dx.doi.org/10.1364/AO.35.006236

[14] Zhang, Z.H., Wang, H.Y., Liu, Z.Q., Huang, M., Liu, F.F., Yu, M.J. and Zhao, B.Q. (2012) Phase Unwrapping Algorithms Based on Fast Fourier Transform. Laser \& Optoelectronics Progress, 49, 62-68. 